\title{
Resolution Models for Energy-Filtered TEM Imaging over Thick Liquid or Amorphous Layers
}

Eduardo Ortega ${ }^{1}$ and Niels de Jonge ${ }^{2}$

${ }^{1}$ INM - Leibniz Institute for New Materials, Saarbrücken, Texas, Germany, ${ }^{2}$ INM - Leibniz Institute for New Materials, Saarbrücken, Germany

The use and implementation of commercial platforms for in situ liquid S/TEM experimentation have resulted in the imaging and analysis of microscopic processes at atomic resolution in either their native liquid environment or even under external stimuli $[1,2]$. However, image resolution under TEM degrades rapidly with the increasing projected thickness of the liquid cell, as the incident electron beam experiences multiple scattering events. The elastic scattering, resulting in beam blurring effect depends on the medium density and object, i.e. nanoparticle, position over the forward direction of the electron beam path. The inelastic scattering, resulting in chromatic blurring is produced by the electron energy spread driven by spherical and chromatic aberrations. These effects can be reduced recording only the electrons that contribute to the image formation at the expected sample thickness. In this work, we demonstrate the fidelity of TEM numerical models to match and predict the resulting achievable resolution for energy filtered TEM (EFTEM) imaging of thick samples.

The theoretical models including elastic- and inelastic scattering, beam broadening, and chromatic aberration to evaluate resolution as function of the electron dose $(\bar{e} D)$ has been laid out in previous work of de Jonge [3]. However, experimental measurements and their expansion to other operational modes such as EFTEM were needed to analyze the different sample configurations found during liquid-, cryo- or in situ TEM. To model the contrast mechanisms found during EFTEM it is necessary to change the two main components affected by the introduction of an energy slit. The first, chromatic blurring, is limited changing the energy spread $(\Delta \mathrm{E})$ by the energy slit width $(\delta \mathrm{W})[3]$. The second, the effective $\bar{e} D$, is reduced following $t / \lambda_{0}=-\ln \left(I_{0} / I_{t}\right)$ which gives the fraction of elastic scattering electrons according to the target thickness $(t)$ and the inelastic mean free path $\left(\lambda_{0}\right)[3,4]$. If the sample composition and geometry is known, relative thickness values $\left(l_{(0)}\right)$ can be used instead to relate the loss of resolution effects between different materials. Our following calculations evaluate Au, C, $\mathrm{SiN}$ and $\mathrm{Si}$ each with $l_{(0)}=13,210,115$ and $152 \mathrm{~nm}$, respectively.

The previous numerical models were validated with experimental data. Our experimental setup consisted of $15 \mathrm{~nm}$-diameter gold nanoparticles (AuNPs) deposited on a Si microchip with a $50 \mathrm{~nm}$-thick SiN window. Images were acquired using a JEOL ARM 200F operated at $200 \mathrm{kV}$ equipped with a charge coupled device (CCD) camera at the exit of an image energy filter (GIF Quantum 963). The camera was used to acquire unfiltered ("TEM") and energy filtered images with $\delta \mathrm{W}=10 \mathrm{eV}$ over a fixed energy loss $\delta \mathrm{E}=40 \mathrm{eV}$ ("EFTEM"). The exposure time allowed $\bar{e} D$ variations from 250 to $0.1 \overline{\mathrm{e}} / \AA^{2}$. As observed in Fig. 1a, imaging was performed at the edge of the $\mathrm{SiN}$ window to measure the effect of sample thickness. During postprocessing, line profiles of the AuNPs (Fig. 1b) were adjusted to a Gaussian fit using a custom Matlab program. From the normalized fit, the spatial resolution is measured from the Gaussian $25-75 \%$ rising edge width $\left(\mathrm{r}_{25-75}\right)$ and the full width at half maximum (FWHM) [5]. On Fig. 1c. the experimental $r_{25-75}$ values and the expected theoretical models are compared. The introduction of the energy window improved the SNR and therefore the observed resolution at thicker regions. Although gold deposited on Si was used in the experiment, these equations can be applied to any material or geometry. As an example, Fig. 1d presents a comparison between carbon and gold nano-objects imaged within a $1 \mu \mathrm{m}$ water layer confined between two SiN windows $(2 \times 50 \mathrm{~nm})$. The combined thickness effect for $\mathrm{H}_{2} \mathrm{O}$ and $\mathrm{SiN}$ accrue to $4 l_{(0)}$ when $l_{\text {water( }(0)}=330 \mathrm{~nm}$ is added into the calculations. The height position $(H)$ of the nanoparticle (at $3 / 4$ of the thickness of the water layer) is added to induce a beam blurring effect. EFTEM becomes useful when the reduced $\bar{e} D$ overcomes SNR limitations, reaching $4.9 \mathrm{~nm}$ around $300 \mathrm{e}^{-/} / \AA^{2}$. A common resolution of $5.8 \mathrm{~nm}$ is reached at $13.1 \mathrm{e}^{-} / \AA^{2}$ by standard TEM and $5.5 \mathrm{e}^{-} / \AA^{2}$ with EFTEM . For the case of carbon vs. water there is no clear benefit of working under 
EFTEM. We will further discuss aspects of experimental setup, operational parameters, and the roll of chromatic aberration TEM imaging to improve spatial resolution during in situ/liquid-cell data acquisition [6].

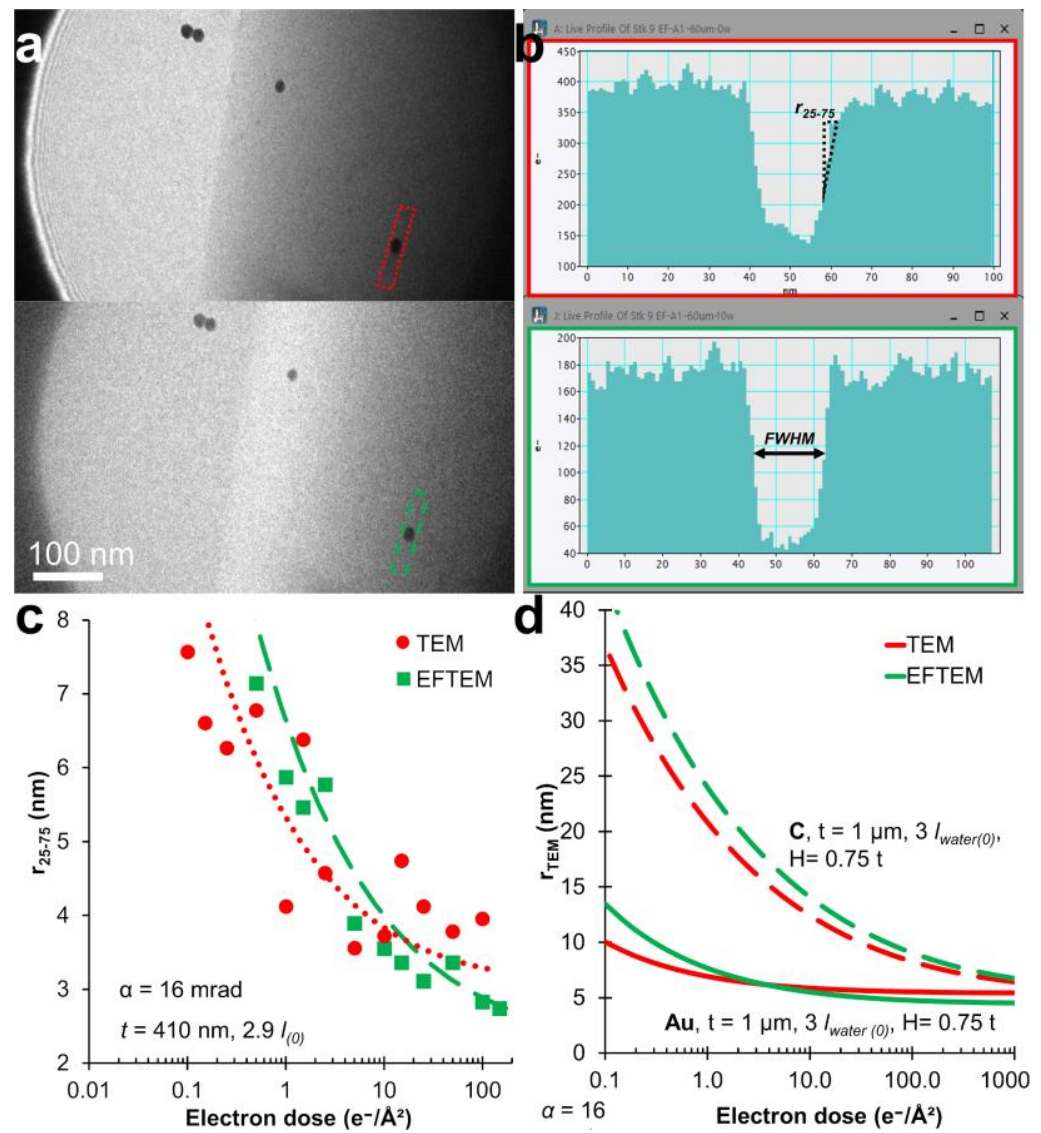

Figure 1. (a) Unfiltered TEM image (top) and filtered EFTEM image (bottom) acquired with an objective aperture equal to $\alpha=16 \mathrm{mrad}$ at $25 \mathrm{e}^{-} / \AA^{2}$. (b) Line profiles for the AuNPs highlighted in (a). The energy window $(\delta \mathrm{W}=10 \mathrm{eV}, \delta \mathrm{E}=40 \mathrm{eV})$ reduced intensity by a factor of 2.5. (c) Comparison of the spatial resolution measurements $\left(\mathrm{r}_{25-75}\right)$ of a AuNP $410 \mathrm{~nm}$ on top of a SiN/Si wedge. Calculated curves were included for the two operational modes (d) Relation between $r_{T E M}$ and $\bar{e} D$ for gold and carbon nanoparticles in $1 \mu \mathrm{m}$ of water between two $50 \mathrm{~nm}$ thick SiN windows, giving an equivalent thickness effect of $4.0 l_{(0)}$, for TEM and EFTEM $(\delta \mathrm{W}=20 \mathrm{eV})$. The particle is located at a height of $3 / 4$ of the thickness of the water layer to induce an additional beam blurring effect.

\section{References}

[1] J Yang et al., Microsc Microanal 25(S2) (2019) p. 1406.

[2] SM Ghodsi et al., Small Methods 3 (2019) 1900026.

[3] N de Jonge, Ultramicroscopy 187 (2018) p. 113.

[4] DS Su, HF Wang and E Zeitler, Ultramicroscopy 59 (1995) p. 181.

[5] N de Jonge, A Verch and H Demers, Microsc Microanal 24 (2018), p. 8.

[6] The authors thank E. Arzt for his support through INM. The research was supported by the German Research Foundation. 